\title{
Nucleosynthesis in neutron-rich ejecta from quark-novae
}

\author{
P. Jaikumar ${ }^{1}$, B. S. Meyer ${ }^{2}$, K. Otsuki ${ }^{3}$, and R. Ouyed ${ }^{4}$ \\ 1 Department of Physics and Astronomy, Ohio University, Athens, OH 45701, USA \\ e-mail: jaikumar@imsc.res.in \\ 2 Department of Physics and Astronomy, Clemson University, Clemson, SC 29634, USA \\ 3 Department of Astronomy and Astrophysics, Enrico Fermi Institute, University of Chicago, Chicago, IL 60637, USA \\ 4 Department of Physics and Astronomy, University of Calgary, 2500 University Drive NW, Calgary, Alberta, T2N 1N4, Canada
}

Received 18 October 2006 / Accepted 23 May 2007

\section{ABSTRACT}

\begin{abstract}
We explore heavy-element nucleosynthesis by rapid neutron capture (r-process) in the decompressing ejecta from the surface of a neutron star. The decompression is triggered by a violent phase transition to strange quark matter (quark-nova scenario). The presence of neutron-rich large $\mathrm{Z}$ nuclei $(40,95)<(Z, A)<(70,177)$, the large neutron-to-seed ratio, and the low electron fraction $Y_{\mathrm{e}} \sim 0.03$ in the decompressing ejecta present favorable conditions for the r-process. We perform network calculations that are adapted to the quark-nova conditions, and which mimic usual $(n-\gamma)$ equilibrium r-process calculations during the initially cold decompression phase. They match to dynamical r-process calculations at densities below neutron drip $\left(4 \times 10^{11} \mathrm{~g} \mathrm{~cm}^{-3}\right)$. We present results for the final element abundance distribution with and without heating from nuclear reactions, and compare to the solar abundance pattern of r-process elements. We highlight the distinguishing features of quark-novae by contrasting it with conventional nucleosynthetic sites such as type II supernovae and neutron star mergers, especially in the context of heavy-element compositions of extremely metal-deficient stars.
\end{abstract}

Key words. dense matter - nuclear reactions, nucleosynthesis, abundances - stars: neutron

\section{Introduction}

The r-process is of fundamental importance in explaining the origin of many heavy neutron-rich nuclei $(A>90-100)$ (Burbidge et al. 1957; Cameron 1957). While the astrophysical conditions that lead to a successful r-process can be estimated, the astrophysical site that can provide these conditions evades positive identification. Recent comprehensive spectroscopic studies of extremely metal-poor stars in the Galactic halo (Hill et al. 2002; Cowan et al. 2002; Sneden et al. 2003), as well as chemical evolution studies (Ishimaru \& Wanajo 1999; Ishimaru et al. 2004; Argast et al. 2004), confirm that a) the r-process is a robust primary nucleosynthetic mechanism and b) core-collapse (type II) supernovae ( $\mathrm{SNe}$ ) are favored over neutron star mergers (NSMs) as its astrophysical site. However, a robust r-process model without parameterization in the neutrino-driven wind of a core-collapse supernova is, thus far, lacking (Qian 2003). Other astrophysical sites explored in the context of a successful r-process include: prompt supernova explosions from small iron cores (Sumiyoshi et al. 2001) or from O-Ne-Mg cores (Wanajo et al. 2003), and neutron star mergers (Freiburghaus et al. 1999a). Although these are promising candidates, they face major theoretical and observational challenges, as outlined below. In this paper, we explore decompressing neutron matter from the surface of a neutron star as an alternative or additional site for r-process nucleosynthesis. Previously, decompression was attributed to tidal disruptions in neutron star-neutron star or neutron star-black hole collisions (Lattimer \& Schramm 1974; Symbalisty \& Schramm 1982; Freiburghaus et al. 1999a), explosion of a neutron star below its minimum mass (Sumiyoshi et al. 1998), or equatorial mass shedding during spin-down of rapidly rotating supramassive neutron stars.
In this work, we consider a new decompression scenario: a violent nuclear-quark phase transition in the core of a neutron star that ejects neutron-rich matter at the surface: the quark-nova (Keränen et al. 2005). Our main aim is to evaluate the quarknova $(\mathrm{QN})$ as a nucleosynthetic site and contrast it with more conventional alternatives, namely type II SNe and NSMs, in the context of observations. We begin by highlighting important observations that can judge relative merits of the astrophysical sites studied so far.

The extremely metal-poor $([\mathrm{Fe} / \mathrm{H}]=-3.1)$ halo giant CS 22892-052 shows an overabundance of r-process nuclei compared to Fe-peak nuclei; e.g., Eu/Fe exceeds the corresponding solar values by factors of 30 or more (Sneden et al. 1996, 2003). However, the relative element abundances in the range $56<Z<82$ (i.e., Ba through $\mathrm{Pb}$ ) in CS 22892-052 follow the solar r-process pattern. This suggests that the (strong) r-process operates largely unchanged over the long history of Galactic chemical evolution, and is associated with a unique astrophysical process. Considerable effort has therefore been invested in detailed modelling of core collapse SNe and NSMs, accompanied by chemical evolution studies, in the hope of explaining the abundance observations and scatter plots of r-process-only elements such as Europium (Eu) (Argast et al. 2000, 2004).

NSMs occur at a much lower rate ${ }^{1}$ than any type of SNe and so face problems in explaining the early onset (at times corresponding to $[\mathrm{Fe} / \mathrm{H}] \approx-2.5)$ of correlations between the ratio of r-process elements [r/Fe] and Fe enrichment (Qian 2000).

\footnotetext{
1 The coalescence rate for binary neutron stars is assumed to range from $3 \times 10^{-4} \mathrm{yr}^{-1}$ to $10^{-6} \mathrm{yr}^{-1}$ (Belczynski et al. 2002) with coalescence times that range from 100-1000 Myr (Portegies et al. 1998; Fryer et al. 1999).
} 
Furthermore, neutron star mergers are unable to satisfactorily explain the associated scatter plots derived from observations of extremely metal-poor halo stars $([\mathrm{Fe} / \mathrm{H}]<-3.0)$ and thick disk stars $(-1.0<[\mathrm{Fe} / \mathrm{H}]<0.2)$, which show that the scatter in $[\mathrm{Eu} / \mathrm{Fe}]$ falls from $\sim 2.0$ dex at early times to $\sim 0.2$ dex at later times (Argast et al. 2004).

Comparative observations of r-element abundance patterns in metal-poor stars such as CS 22892-052 and CS 31082-001 $([\mathrm{Fe} / \mathrm{H}]=-2.9)$ suggest that elements from $\mathrm{Ba}$ and above $(A>$ 130) follow the solar r-element pattern while those below $A \sim$ 130 fall below the extension of this pattern. A similar conclusion is drawn from the behaviour of the strontium to barium ([Sr/Ba]) ratio in a comparison of r-process rich and r-process poor stars (Truran et al. 2002). An explanation of this fact based on corecollapse SNe may originate in differing progenitor masses (Qian $2004)^{2}$. Intriguingly, a successful match to the solar r-process pattern (particularly around the 3rd r-process peak $(A \sim 195)$ ), produced by $\mathrm{SNe}$ events associated with the low-mass progenitors, seems to require an unusually massive $\left(M \sim 2.0 M_{\odot}\right)$ neutron star mass (Otsuki et al. 2000; Sumiyoshi et al. 2000). Such heavy neutron stars with canonical radius $(R \sim 15 \mathrm{~km})$ would have large central densities provided the high density equation of state is not too stiff. They may be prone to a nuclear-quark deconfinement transition at a later stage.

Data from metal-poor stars point toward the distinct possibility of multiple r-process sites with varying astrophysical environments. A conclusive association of r-processing with core-collapse $\mathrm{SNe}$ events awaits further spectral identification of newly-synthesized r-process nuclei (Mazzali \& Chugai 1995) and detection of gamma-rays from decays of r-process nuclei (Meyer \& Howard 1991; Qian et al. 1998).

Aside from the challenges posed by abundance observations, there are hurdles on the theoretical side as well. Progress in the modelling of type II SNe and $\gamma$-ray bursts has led to the viable scenario of "neutrino-driven winds" from nascent neutron stars (Woosley et al. 1994; Takahashi et al. 1994; Qian \& Woosley 1996; Wanajo et al. 2001; Otsuki et al. 2000; Thompson et al. 2001; Cardall \& Fuller 1997), where presumably the r-process is successful if model parameters are chosen appropriately. Unfortunately, values of model parameters extracted from corecollapse supernova simulations, such as the entropy per baryon, electron fraction and expansion timescale do not reproduce the observed solar abundance of r-process elements. One possible implication of this is that the r-process has an additional site with different neutron exposure. The prompt-explosion model of a collapsing O-Ne-Mg core does reproduce the solar-like $\mathrm{r}$ abundance pattern (Wanajo et al. 2002, 2003) but an artificial enhancement of the shock-heating energy is needed to obtain the requisite physical conditions for a successful r-process.

In neutron star mergers, the amount of mass ejected depends strongly on the poorly-known high-density equation of state, with stiffer equations of state predicting larger mass loss (Rosswog et al. 2001). General relativistic considerations for the system dynamics, the sensitivity of the network calculations to the electron fraction (which actually has a spread in neutron star crusts), and the effects of neutrino transport on the former during the coalescence phase present sources of uncertainty. Furthermore, a universal description of all relevant nuclear data

\footnotetext{
2 Alternate sites for heavy-element r-processing include accretioninduced collapse (AIC) of a white dwarf into a neutron star in a binary, outflows in gamma-ray bursts (Surman et al. 2006) and collapse of hybrid stars of white dwarf dimensions (Marranghello \& de Freitas Pacheco 2005).
}

that would ensure good systematics remains out of reach, until reaction cross-sections and decay half-lives of many unstable exotic nuclei involved in the r-process network can be measured accurately in rare-isotope experiments.

To overcome these challenges and explain the solar system composition in a satisfactory way requires new astrophysical input as well as better nuclear systematics. In this work, we wish to focus on the possibilities and implications of the decompression scenario with input parameters determined by the specifics of a quark-nova. This is not the first work to investigate r-process nucleosynthesis in decompressing neutron matter. Meyer (1989) and Goriely et al. (2004) have studied the r-process in the presence of exotic nuclei formed in matter resulting from the decompression of initially cold neutron star matter (Lattimer et al. 1977), although no specific mechanism for decompression was proposed $^{3}$. They found that improvements in radiative neutron capture rates by exotic nuclei close to the neutron drip line ${ }^{4}$ and fission probabilities of heavy neutron-rich nuclei lead to good agreement between the predicted and the solar abundance pattern for $A \geq 140$ nuclei (see Fig. 2 in Goriely et al. 2004). Those studies are the first consistent calculation of the nucleosynthetic composition of dynamically ejected material from the neutron star surface.

Interestingly, the initial conditions for decompression considered by Goriely et al. (2004) for a reasonably successful reproduction of the heavy-element r-process pattern are almost characteristic of the quark-nova explosion and its ejecta. After explaining the quark-nova scenario in Sect. 2, we set up the decompression dynamics in the setting of a quark-nova in Sect. 3. In Sect. 4, we present results from our network calculations of the decompression scenario, and compare them with the solar r-process pattern. We consider cases of slow and fast decompression with and without nuclear heating from $\beta$-decays. In Sect. 5, we compare and contrast the QN with NSM and type II SNe as astrophysical sites for the r-process, particularly in the context of Galactic chemical evolution. We address observational signals of the r-process event such as $\gamma$-rays from the radioactive decay of heavy r-process nuclei. Our conclusions and a discussion of certain aspects that have not been covered in this work, and which require further study, are presented in Sect. 6.

\section{Quark-nova: nuclear-quark phase transition}

It has long been thought that the center of neutron stars may be dense enough that nuclear boundaries dissolve and a phase transition to quark matter occurs (Itoh 1970; Bodmer 1971). Up and down quarks would then preferentially convert to strange quarks (other heavier flavors can be neglected) in order to reduce Pauli repulsion by increasing flavor degeneracy. This idea was formally stated as a conjecture (Witten 1984) that strange quark matter is the true ground state of strongly interacting matter at zero pressure. Even if true only at finite pressure (Farhi \& Jaffe 1984), its consequences for neutron stars are sufficiently intriguing that several possibilities for such a phase conversion have been examined (Alcock et al. 1986). One attractive possibility is that nuclear matter undergoes a phase transition to two flavor (up and down) quark matter, which then undergoes weak

\footnotetext{
3 Alternate mechanisms for ejection of initially cold, decompressed neutron star matter were mentioned in the introduction.

4 In addition to the intermediate compound nucleus, direct electromagnetic transition to a bound final state, which is important for neutron-rich exotic nuclei, was included.
} 
equilibrating reactions to form three flavor quark matter (Olesen \& Madsen 1991; Cheng \& Dai 1996). The advantage is that this does not require the improbable simultaneous and spontaneous conversion (via weak interactions) of several neutrons within a small volume. In addition, the Coulomb-barrier-free absorption of neutrons can enlarge the quark phase. If Witten's conjecture holds, the whole neutron star is essentially converted to strange quark matter, thus forming a quark star.

There are various ways in which this conversion can be triggered. A rapidly rotating neutron star is slowed down principally by magnetic braking (and additionally by energy loss through gravitational waves), thereby reducing the centrifugal force and increasing the central pressure. The probability of triggering a first order transition by forming a tiny lump of two flavor quark matter (quantum nucleation) is exponentially sensitive to the central pressure (Bombaci et al. 2004). This makes the transition much more likely as the star spins down. Material accumulated from a fallback disk around a cooling neutron star can also force the conversion. Even the Bondi accretion rate of $10^{21} \mathrm{~g} / \mathrm{s}$ from the interstellar medium can lead to a mass increase of up to $0.1 M_{\odot}$ within a million years which can be sufficient to compress the star beyond the minimum density for the phase transition. Microscopic pathways for the phase conversion include the clustering of $\Lambda$-baryons at high density and seeding by energetic cosmic neutrinos or strangelets (Alcock et al. 1986). Deconfinement of quarks can also occur as early as the protoneutron star stage after a supernova explosion if the central density is large enough. However, in this case, neutrino trapping in the hot and dense interior can push the transition density higher, delaying the collapse to the point where a black hole might be formed instead (Prakash et al. 1997).

In this work, we do not address in detail the mechanism by which the conversion is triggered, but clearly it is important to determine the likelihood of such a conversion, since it is linked to the frequency of the event that generates the r-process elements in our model calculation. Yasutake et al. (2005) have determined that the evolutionary transition from rapidly rotating neutron stars to strange stars due to spin down can lead to an event rate of $10^{-4}-10^{-6}$ per year per Galaxy. A more comprehensive analysis employing different equations of state (the simplistic Bag model was employed in Yasutake et al. 2005) and a variety of initial conditions at neutron star birth finds a similar event rate (Staff et al. 2006). In light of the limited work on the above topic, we will assume that spin-down is responsible for the phase transition.

In the quark-nova picture, (Ouyed et al. 2002; Keränen \& Ouyed 2003; Keränen et al. 2005) the core of the neutron star that undergoes the phase transition to the quark phase shrinks in a spherically symmetric fashion to a stable, more compact strange matter configuration faster than the overlaying material (the neutron-rich hadronic envelope) can respond, leading to an effective core collapse. The core of the neutron star is a few kilometers in radius to begin with, but shrinks to $1-2 \mathrm{~km}$ in a collapse time of about $0.1 \mathrm{~ms}$ (Lugones et al. 1994). The gravitational potential energy thus released is converted partly into internal energy (latent heat of phase transition) and partly into outward propagating shock waves which impart kinetic energy to the material that eventually forms the ejecta. The temperature of the quark core thus formed rises quickly to about $10 \mathrm{MeV}$ since the collapse is adiabatic rather than isothermal (Gentile et al. 1993). In Keränen et al. (2005), the core bounce was neglected, and neutrinos emitted from the conversion to strange matter were assumed to transport the energy into the outer regions of the star, leading to mass ejection. With neutrino-driven mass ejection, most of the neutrinos that can escape the core lose their energy to the star's outer layers of neutron matter in the form of heat. Consequently, mass ejection is limited to about $10^{-5} M_{\odot}$ for compact quark cores of size (1-2) km. More importantly, the neutrinos raise the electron fraction in the ejected layers by converting neutrons to protons, with a resultant $Y_{\mathrm{e}} \sim 0.5$. This is unfavorable for producing the distinct peaks of the r-process. In fact, simulations of core collapse induced by a nuclear-quark phase transition (Fryer \& Woosley 1998) have shown that for $Y_{\mathrm{e}} \sim 0.5$, the bulk of the ejecta is helium, although some iron and r-process elements are produced.

A more attractive possibility is that of core bounce, accompanied by outward propagating shocks that can impart sufficient kinetic energy to the outer layers, including the crust of the star. Since most of the neutrinos will remain trapped within the advancing front of strange matter as it engulfs the star (Keränen et al. 2005), the $Y_{\mathrm{e}}$ of the ejected material is small, and allows for an effective r-process. It is important to note that only a fraction of neutrinos from weak processes in the $(u, d, s)$ core can escape before the entire star converts to strange matter. This assumes that the $(u, d, s)$ contamination front moves at supersonic speeds (detonation) rather than by combustion (deflagaration). Studies by Horvath \& Benvenuto (1998) confirm that in a detonation regime, the star would rapidly convert to quark matter within a time $0.1 \mathrm{~ms}$. More recent work, assuming realistic quark matter equations of state, argues for strong deflagration (Drago et al. 2005), but this too may be preceded by a compression shock (Lugones et al. 1994) in the nuclear region that can expel surface material.

Although an accurate study of the shock propagation and the advancing quark conversion front would require detailed modelling of neutrino transport, as a first approximation, we neglect neutrino transport at core bounce, since only about $1 \%\left(10^{51} \mathrm{erg}\right.$ out of $10^{53}$ erg released in the phase conversion) of the energy leaves the core in the form of neutrinos over typical timescales of mass ejection (due to long diffusion times of neutrinos in the hot quark core). The ejected mass fraction is expected to be larger (about $10^{-2} M_{\odot}$ ) than the previous case which neglected core bounce. This is analogous to the mechanism of mass ejection from phase transitions in a supernova as considered by Fryer \& Woosley (1998), which can cause considerably more mass ejection due to an outgoing shock wave from the supersonic collapse of the strange matter core. The main difference from their work is that in a quark-nova, the neutrinos remain trapped in the hot quark core as it grows and engulfs the star, and they cannot increase the $Y_{\mathrm{e}}$ of the ejected matter significantly (Keränen et al. 2005).

\section{Decompression of the ejecta}

In a quark-nova, the decompression is powered by shock waves, and it is possible that shock heating of the ejecta up to temperatures of $10^{10}-10^{11} \mathrm{~K}$ increases the $Y_{\mathrm{e}}$ due to positron capture by the neutron-rich material if it is not expanding faster than weak interaction timescales. Thus, a rapid ejection of the material is essential for efficient r-process nucleosynthesis. Apart from being neutron-rich, the QN ejecta is also rich in exotic nuclei with $56<A<118$ which come from the outermost layers of densities at and below neutron drip (Baym et al. 1975b). Thus, electrical neutrality demands that there should be a spread in $Y_{\mathrm{e}} \approx$ 0.03-0.2 in the crust. Since our calculations are performed for a particular initial $Y_{\mathrm{e}}$, our results are to be interpreted as corresponding to a mass-averaged $\left\langle Y_{\mathrm{e}}\right\rangle$, which depends on the ejected 
mass. We comment on this sensitivity of the yield to ejected mass (through $\left\langle Y_{\mathrm{e}}\right\rangle$ ) at the end of Sect. 4.

It is also possible that the initial temperature of the ejecta $\left(T_{9}^{i}\right)$ is variable, depending on whether the transition to quark matter happens within seconds of the protoneutron star formation (in which case $\left.T_{9}^{i} \sim 10\right)$ or much later $\left(T_{9}^{i} \sim 0.1\right.$ or smaller). The escaping neutrinos will also increase the temperature of the neutron-rich material. As shown by Meyer (1989) and Rosswog et al. (2000), the conditions for nucleosynthesis in the decompression scenario are largely independent of the choice of initial temperature within a range $T_{9} \approx 0-1$, since in all cases, the expanding material heats up to r-process like temperatures by $\beta$-decays, so we choose $T_{9}^{i} \approx 1$ or smaller as our initial temperature. Since mass ejection is largely unaffected by internal heating from neutrino absorption (Rosswog et al. 1999), the temperature evolution of the ejecta is subsequently determined by adiabatic expansion and nuclear reactions (specifically $\beta$-decays when they become allowed). The dynamical $(n-\gamma)$ equilibrium calculations employed here allow us to study this temperature evolution.

\section{Initial conditions for decompression}

For the matter that is eventually ejected from the neutron star, the compositions above and below neutron drip, as well as the nuclear models used, are taken from the work of Meyer \& Schramm (1988) and Meyer (1989). This improves upon the results of the compressional liquid drop model pioneered by Baym et al. (1971a), and Lattimer et al. (1985) by including nuclear deformations and shell effects. For the equation of state for dense, $\beta$ - equilibrated matter described in Meyer (1989) and Goriely et al. (2004), the composition at $\rho \simeq 10^{14} \mathrm{~g} / \mathrm{cc}$ is characterized by an initial electron fraction $Y_{\mathrm{e}}=0.03$, corresponding to a Wigner-Seitz cell made by a $Z=36, N=157$ nucleus. However, our network calculations are performed with $Y_{\mathrm{e}}$ as a parameter for reasons explained previously. The details of the computational methods are given in the following section.

The expansion of this dense ejecta (including $\beta$-decays) is followed down to neutron drip density using the network calculation of Meyer (1989), where the matter ends up distributed over a wide range of elements from $Z \sim 40-70$. At this stage, the full r-process reaction network is coupled to obtain the final abundance distribution, as well as the evolution of temperature, entropy and electron fraction. An important parameter other than $Y_{\mathrm{e}}$ is the expansion timescale for the ejecta, which is the time taken to evolve from a density of $\rho \simeq 10^{14} \mathrm{~g} / \mathrm{cc}$ to $\rho \simeq 10^{11} \mathrm{~g} / \mathrm{cc}$. Assuming that the ejecta is on an escape trajectory and expands as a multiple of the free-fall timescale in a spherically symmetric, non-interacting manner, we obtain a timescale on the order of $0.01-0.1 \mathrm{~ms}$.

Since neutrinos are trapped within the quark core on account of their long diffusive timescales of $0.1 \mathrm{~s}$, the expansion is fast enough that $Y_{\mathrm{e}}$ is not much changed by neutrinos, as explained previously. Nevertheless, the results are expected to be sensitive to the expansion timescale, so we vary this parameter from 0.01-1 ms to test the sensitivity of our abundance distribution to this parameter. In their calculations, Goriely et al. (2004) find that the final abundance pattern can be significantly different from solar for expansion timescales $\tau<3 \mathrm{~ms}$, since not all neutrons are captured. In the quark-nova scenario, the rapid conversion to strange quark matter constrains us to explore a bounded range of timescales $0.01<\tau(\mathrm{ms})<0.1$. For the sake of comparison with results in Goriely et al. (2004), we also perform one set of calculations with $\tau \sim 1 \mathrm{~ms}$, which would correspond to a slow expansion, but is not likely to be the case in the quark-nova scenario. The following section contains the results for heavyelement nucleosynthesis in the decompression scenario. A comparison to the solar abundance pattern in each case is also presented.

\section{4. r-nuclei abundance distribution}

We use the Clemson University nucleosynthesis code (Jordan $\&$ Meyer 2004) to explore the nuclear yields that result from the decompression of matter ejected from a quark-nova. This particular network includes species from neutrons and protons up to uranium and all isotopes between the proton-drip line to several nuclides beyond the neutron-drip line for each element. We choose to extend the network beyond the neutron-drip line because some of our expansions reach such low temperatures that neutron captures are able to branch across neutron-unstable species in some cases. The last species in the network $Z=92$, $A=276$ is assumed to fission into two fragments-one with $Z=$ 40 and one with $Z=52$. This provides strong fission cycling of the r-process flow in certain cases.

We follow the matter beginning at $Y_{\mathrm{e}}=0.03, T_{9}=1$ and an entropy per nucleon $s / k_{\mathrm{B}}=1$. We compute the temperature and density history of the material by imagining a spherical chunk of matter of radius $R$ decompressing due to its own pressure $P$. In the absence of gravity (free fall), the momentum equation, under the assumption of spherical symmetry, reads

$\frac{\mathrm{d} v}{\mathrm{~d} t}=-\frac{1}{\rho} \frac{\partial P}{\partial r}$

where $v$ is the velocity of a radial shell, $\rho$ is the matter density, and $r$ is the radial coordinate of one of the shells. For simplicity, we assume a uniform density in any chunk of expanding matter (at a particular instant) so that

$\frac{\rho}{\rho_{0}}=\left(\frac{R_{0}}{R}\right)^{3}$,

where $\rho_{0}$ and $R_{0}$ are the density and and radius of the chunk at the beginning of the calculation. We approximate Eq. (1) as

$\frac{\mathrm{d}^{2} R}{\mathrm{~d} t^{2}}=\frac{\alpha}{\rho} \frac{P}{R}=\alpha \frac{c_{\mathrm{s}}^{2}}{R}$,

where $\alpha$ is a constant that accounts for deviations from our simple model while also determining the expansion timescale, and $c_{\mathrm{s}}$ is the sound speed. The pressure gradient causes the chunk of matter to accelerate outwards. This increases the radius, decreases the density and pressure and hence, the acceleration. This means that after an initial acceleration, the material tends to reach a coasting speed, which is typically a few percent the speed of light in our calculations.

We compute the pressure from routines available in the nucleosynthesis code, as described in Meyer \& Brown (1997). For each timestep in the calculation, we use Eq. (3) to find the radius of the chunk based on information from the previous timestep. We compute the density from Eq. (2). We then guess the temperature, solve the nuclear network for the changes in the abundances over the timestep, compute the change in the entropy due to nuclear reactions, and then invert the entropy to find the temperature. If the resulting temperature differs from our guess, we modify our guess and repeat the procedure until we find agreement between our guess and the resulting temperature. We then proceed to the next step. 


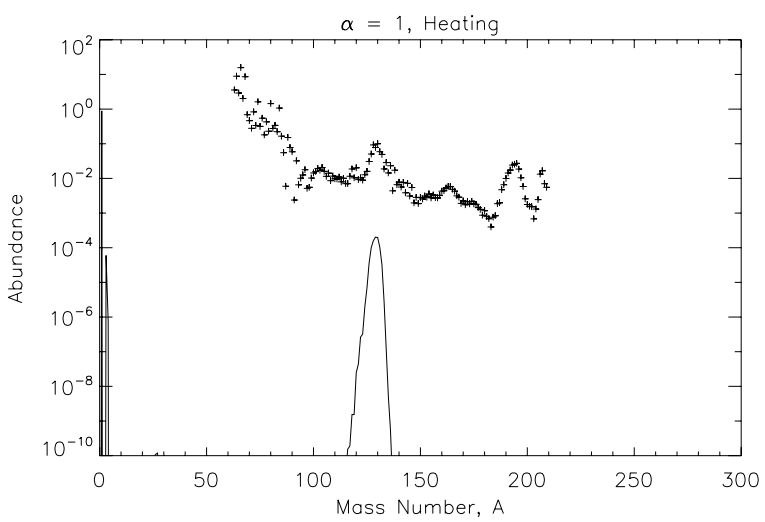

Fig. 1. The final abundance distribution as a function of mass number for the $\alpha=1$ calculation in the case in which heating from nuclear reactions was included. (See text below for details).

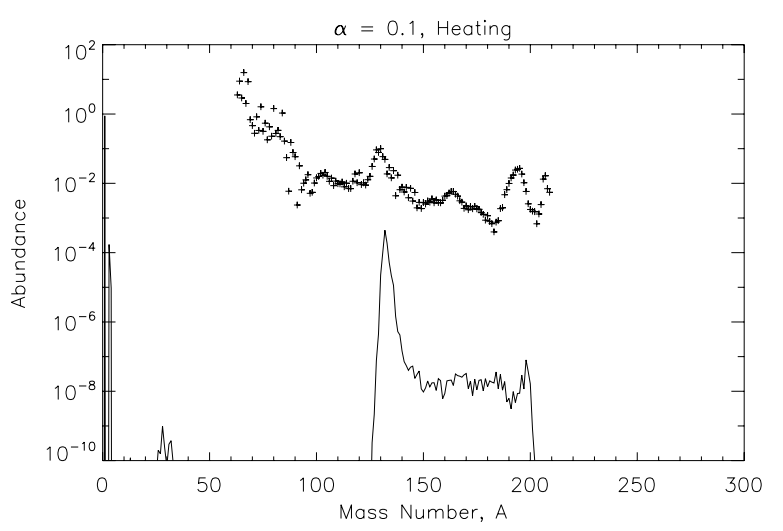

Fig. 2. The final abundance distribution as a function of mass number for the $\alpha=0.1$ calculation in the case in which heating from nuclear reactions was included.

Nuclear reactions out of equilibrium generate entropy and consequently, lead to heating of the material during the expansion. We assume any energy released by reactions is fully deposited locally. There is a difficulty with this assumption. The primary heating in our calculations comes from beta decays. A significant fraction (typically $\sim 60 \%$ for the neutron-rich heavy nuclei in the r-process) of the energy from a beta decay is released as a neutrino, which would escape the chunk without depositing its energy. We do not yet have the details of the relative energy carried away by neutrinos for all the nuclei in the network; thus, to explore this issue, which turns out to have significant consequences for our results, we study two extemes, namely, one in which we assume all energy generated by nuclear reactions was deposited locally and one in which we neglect energy released by the reactions. The former case certainly overestimates the entropy generation by nuclear reactions because it neglects neutrino losses. In the latter case, the entropy during the expansion is constant.

We focus on four particular decompression calculations. The calculations have $\alpha=1$ (corresponding to an expansion timescale $\tau \sim 0.01 \mathrm{~ms})$ or $\alpha=0.1(\tau \sim 0.05 \mathrm{~ms})$ and either include full or zero energy deposition from the nuclear reactions. $R_{0}=10 \mathrm{~m}$ and $\rho_{0}=10^{14} \mathrm{~g} / \mathrm{cc}$ in all cases. The final abundances resulting from these calculations are shown as a function of mass number $A$ in Figs. 1-4. In each case, for comparison, the observed solar r-process abundances (Kappeler et al. 1989) are shown arbitrarily scaled as plus signs.

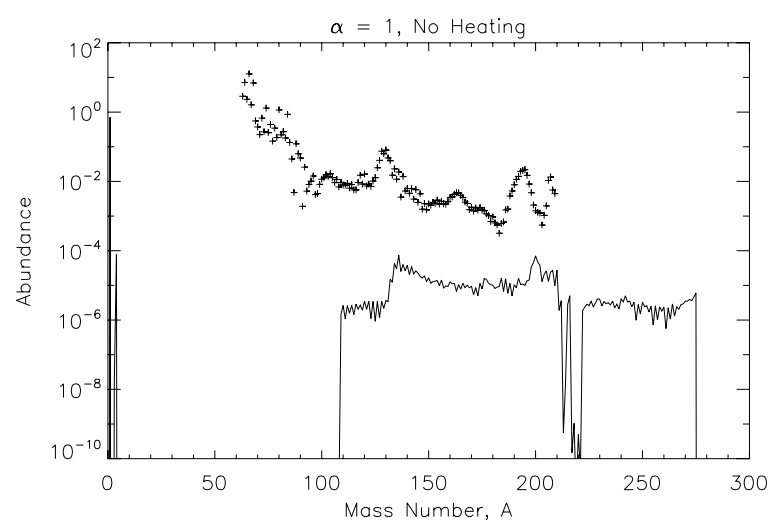

Fig. 3. The final abundance distribution as a function of mass number for the $\alpha=1$ calculation in the case in which heating from nuclear reactions was not included.

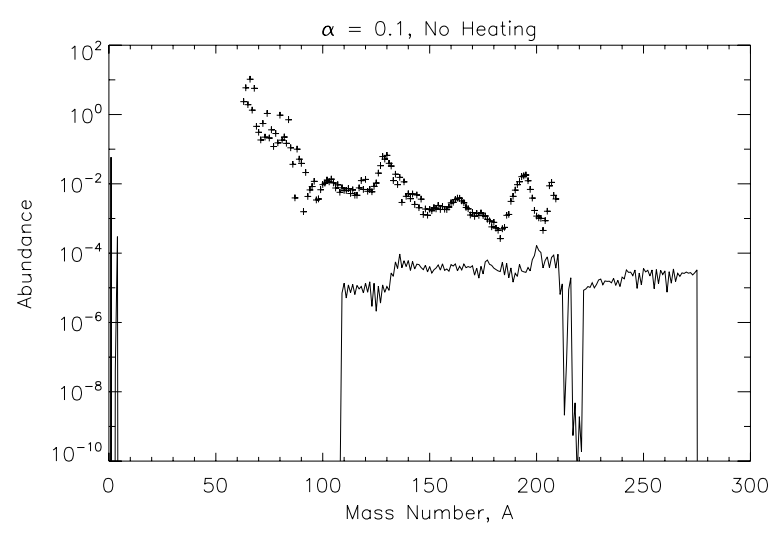

Fig. 4. The final abundance distribution as a function of mass number for the $\alpha=0.1$ calculation in the case in which heating from nuclear reactions was not included.

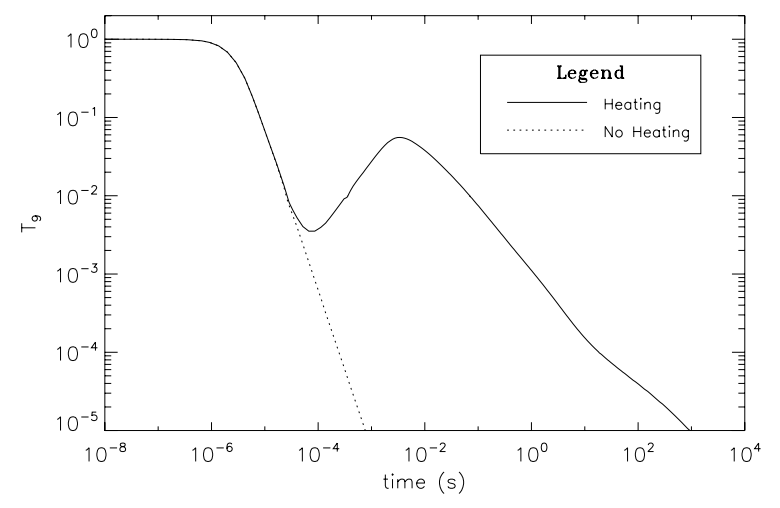

Fig. 5. The temperature as a function of time for the $\alpha=1$ expansion in the cases in which heating from nuclear reactions is included (Heating) or not (No Heating).

sensitivity to heating: As is apparent, calculations that neglect heating from nuclear reactions reach higher mass nuclei. The reason is evident from Figs. 5 and 6, which show the timedependence of the temperature for the various calculations. When heating is included, the temperature initially falls but then begins to rise beginning around $10^{-4} \mathrm{~s}$ as some of the nuclei begin to beta decay. The matter heats up, and the temperature peaks at about $10^{-2} \mathrm{~s}$. At this time, the nuclei have reached the $N=82$ closed shell, and the extra binding forces the r-process path closer to beta stability to the so-called "waiting-point" nuclei where beta rates are slower. The heating thus becomes less 


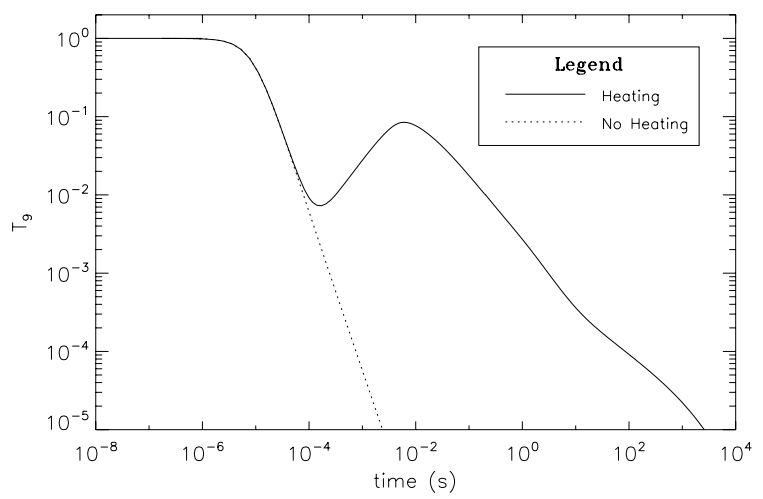

Fig. 6. The temperature as a function of time for the $\alpha=0.1$ expansion in the cases in which heating from nuclear reactions is included (Heating) or not (No Heating).

effective. In fact the slow beta decays at the waiting-point nuclei hold up the flow so much that the rapid expansion allows the neutron-capture reactions to freeze out before consuming the neutrons, and the final abundance distribution is centered on the $N=82$ closed shell. This results in an r-process abundance distribution peaked near $A=130$.

If heating is not included, however, the temperature quickly falls to such low temperatures that neutron disintegration reactions become ineffective. The r-process flow pushes beyond the r-process waiting-point nuclei to isotopes with larger beta decay rates. The r-process flow can then reach higher nuclear mass. The rapid expansion still causes the matter to freezeout before all neutrons are absorbed into nuclei, nevertheless, there is considerable fission cycling. Remarkably, the final r-process abundance pattern does not look like the solar pattern. Because the r-process flow pushes neutron rich of the waiting-point nuclei, the extra binding of the closed neutron shells does not impose itself on the final abundance distribution. Interestingly, the slower expansion with $\alpha=0.1$ does not look significantly different from the $\alpha=1$ case. The flow does reach slightly higher mass in the case with full heating because some nuclei are able to leak past the $N=82$ closed shell before neutron-capture reactions freeze out. Nevertheless, the abundance distribution is still dominated by the $A \approx 130$ peak. It is clear that for $\alpha$ not too different from unity, the degree of energy deposition has a larger effect than the expansion rate.

We investigate a more realistic treatment of heating by running a calculation assuming only $50 \%$ of the energy released by nuclear reactions is deposited locally (to account for energy lost by neutrinos). The final abundance pattern shown in Fig. 7 is little different from that in the full heating calculation shown in Fig. 1. Although the expansion with half energy deposition reheats to a lower temperature than the calculation with the full heating, the resulting temperature is enough to allow disintegration reactions to prevent the flow from bypassing the waiting point nuclei.

sensitivity to $\tau$ : The r-process yield is quite sensitive to $\tau$. To test this sensitivity, we study cases with $\alpha=0.01(\tau \sim 0.1 \mathrm{~ms})$ and $\alpha=0.0001(\tau \sim 1 \mathrm{~ms})$ in which we allow $50 \%$ of the energy released by nuclear reactions to escape from the matter. The initial conditions in the latter case are similar to the choice made in Goriely et al. (2004). The final abundance distribution is shown in Figs. 8 and 9. The relatively slow expansion in these cases allows the nuclear flow to pass the $N=82$ waiting point nuclei

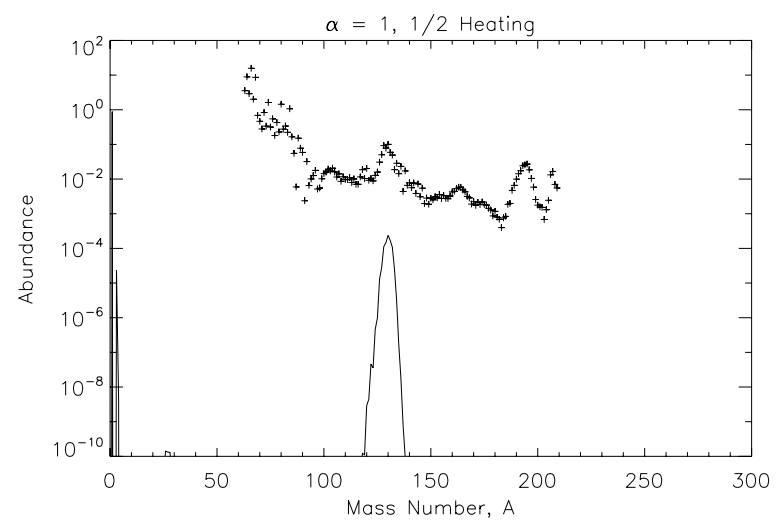

Fig. 7. The final abundance distribution as a function of mass number for the $\alpha=1$ calculation in the case in which $50 \%$ of the energy released by nuclear reactions was assumed to be lost from the matter.

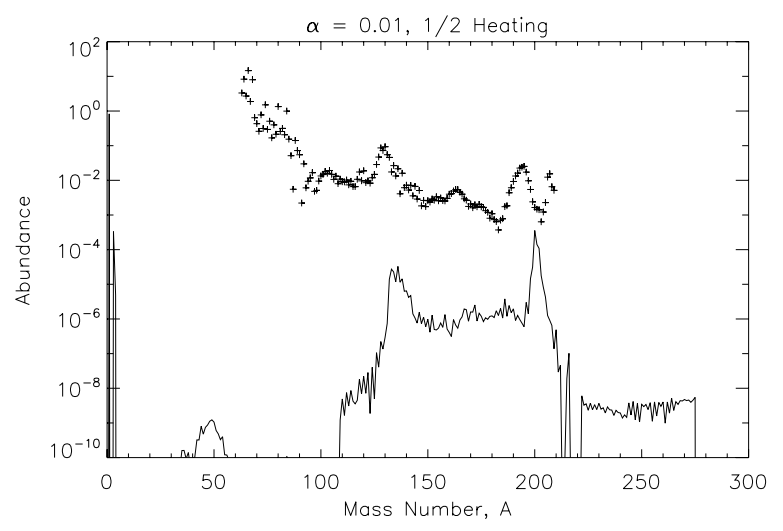

Fig. 8. The final abundance distribution as a function of mass number for the $\alpha=0.01$ calculation and 50\% local energy deposition from nuclear reactions.

before neutron-capture reactions freeze out. These expansions are the most promising in terms of reproducing the heavy solar r-process distribution, as concluded by Goriely et al. (2004). Figures 7-9 clearly portray the strong sensitivity of the yield to the expansion timescale $\tau$, which scales as $1 / \sqrt{\alpha}$ (Eq. (3) of Sect. 4) in our simplified dynamical model of pressure-driven expansion. Smaller $\alpha$ 's imply a slower expansion. In the absence of a hydrodynamical model for the explosion, we have explored a plausible range of timescales $0.01<\tau(\mathrm{ms})<0.1$ by demanding that the mass ejection be completed before the entire star converts to quark matter. The principal effect of a large expansion timescale is that it counteracts the effect of heating from $\beta$-decays which halts the flow at the 2 nd r-process peak and inhibits further neutron capture. A slower expansion can allow the flow to break past the 2 nd peak before all neutrons freeze out. It is clear that the final abundance distribution is quite sensitive to the exact nature of the dynamical trajectory of the expansion and that detailed models that include a full treatment of the reheating from nuclear reactions will be needed to further elucidate the r-process in decompressing matter.

sensitivity to $Y_{\mathrm{e}}$ : Since we lack a dynamical picture of the quark-nova at present, the amount and composition of ejected material is subject to uncertainty. Prior estimates of mass ejection by a quark-hadron phase transition suggest values ranging from 0.001 solar mass to 0.1 solar mass (Fryer \& Woosley 1998). The $Y_{\mathrm{e}}$ will be different in each case depending on the ejected 


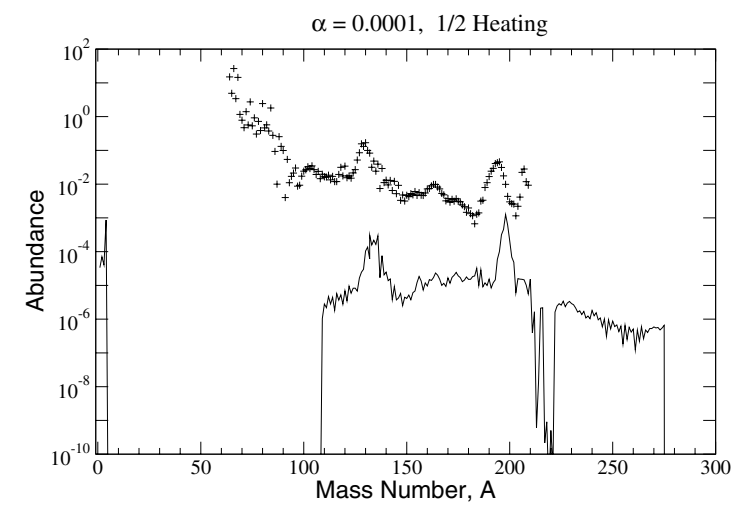

Fig. 9. The final abundance distribution as a function of mass number for the $\alpha=0.0001$ calculation and 50\% local energy deposition from nuclear reactions.

mass. Therefore, we study the dependence of the yield pattern on a mass-averaged electron fraction $\left\langle Y_{\mathrm{e}}\right\rangle$, given by

$\left\langle Y_{\mathrm{e}}\right\rangle=\frac{\int_{R_{\mathrm{c}}}^{R_{0}} Y_{\mathrm{e}}(\rho) \rho \mathrm{d} V}{\int_{R_{\mathrm{c}}}^{R_{0}} \rho \mathrm{d} V}$

where $R_{\mathrm{c}}$ is chosen to give a particular total ejected mass (for the density profile as described below), and $R_{0}=10 \mathrm{~km}$.

To determine $\left\langle Y_{\mathrm{e}}\right\rangle$, we choose the density profile of the neutron star as given in Lorenz et al. (1993). This profile coresponds to the BPS equation of state (Baym et al. 1975b) at low density, matched to the BBP equation of state (Baym et al. 1971a) at densities upto nuclear saturation density. We focus only on 0.01 , 0.001 solar mass ejecta since there is considerable uncertainty for larger ejected mass coming from the high density equation of state. We also study $10^{-5}$ solar mass ejecta as an aside although such a low value is not feasible for the energetics of a quarknova. Using a parameterized formula for the density dependence of $Y_{\mathrm{e}}$ extracted from Table 1 in Meyer (1989), we find the massaveraged $Y_{\mathrm{e}}$ 's to be: $\left\langle Y_{\mathrm{e}}\right\rangle=0.03$ (0.01 solar mass), $\left\langle Y_{\mathrm{e}}\right\rangle=0.09$ (0.001 solar mass), $\left\langle Y_{\mathrm{e}}\right\rangle=0.12\left(10^{-5}\right.$ solar mass $)$ and perform r-process calculations for each of these cases. The following trends for the $\left\langle Y_{\mathrm{e}}\right\rangle$-dependence of the yield (with other parameters $\tau$ and amount of heating fixed) are apparent from Fig. 10 which shows results for the smallest and largest $\left\langle Y_{\mathrm{e}}\right\rangle$ :

1) The yield of the actinide elements relative to lighter elements increases as $\left\langle Y_{\mathrm{e}}\right\rangle$ increases.

2) The position and shape of the second r-process peak is robust in all cases, while the third peak becomes less prominent and less stable with respect to the 2 nd peak as $\left\langle Y_{\mathrm{e}}\right\rangle$ increases.

3 ) Smaller peaks appear around mass numbers $A \sim 44$ (mainly from heavy $\mathrm{Ca}$, Ar and $\mathrm{Ti}$ isotopes) and $A \sim 80$ (mainly from $\mathrm{Se}$ and $\mathrm{Kr}$ and their isotopes) as $\left\langle Y_{\mathrm{e}}\right\rangle$ increases.

These features show that in the quark-nova scenario, as in more conventional sites of the r-process, the 2 nd and the 3 rd r-process peak form under very different conditions of neutron exposure. The 2nd peak does not set very strict limits on the neutron exposure or the energetics of the quark-nova, but the 3rd peak requires significantly more mass ejection and smaller $\left\langle Y_{\mathrm{e}}\right\rangle$, which implies a more energetic explosion. For the case of slow expansion that we studied in these runs, the large neutron-to-seed ratio for small $\left\langle Y_{\mathrm{e}}\right\rangle$ depletes material beyond the 3rd peak due to strong fission cycling and depletes material below the 2 nd peak due to rapid neutron capture. This depletion effect is weakened as we go to higher $\left\langle Y_{\mathrm{e}}\right\rangle$ 's, hence the appearance of the

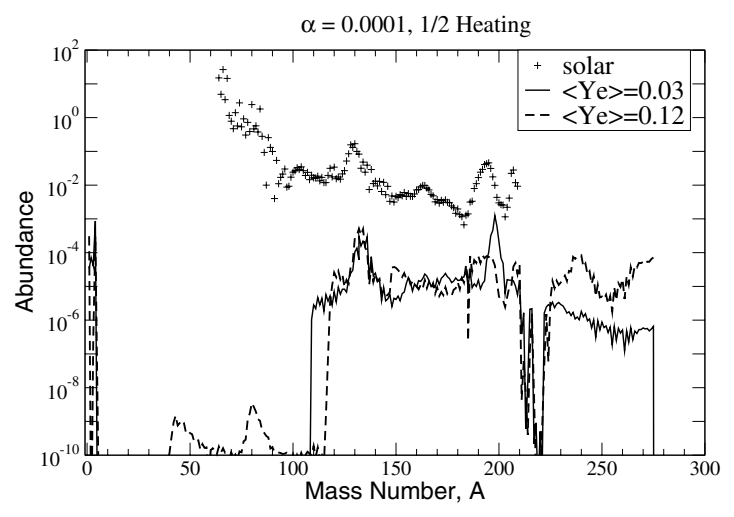

Fig. 10. The final abundance distribution as a function of mass number for the $\alpha=0.0001$ calculation, 50\% local energy deposition from nuclear reactions, and $\left\langle Y_{\mathrm{e}}\right\rangle$ 's as indicated.

smaller peaks, including the expected 1st r-process peak around Selenium 80. The dependence on $\left\langle Y_{\mathrm{e}}\right\rangle$ is therefore quite severe and deserves further study to constrain the energetics of, and element production in, the quark-nova. A coupled hydrodynamic and nucleosynthetic description of the quark nova needs to be done to better constrain the range of $Y_{\mathrm{e}}$ and $\tau$.

\section{Quark-nova as an r-process site}

As observed in the results of the previous section, decompressing neutron matter can be a site for heavy element production. Once the abundance pattern from such an event has been generated, it is natural to turn to the broader astrophysical questions of galactic enrichment by these events, and consistency with observations detailed in the introduction. Though we do not tackle this issue quantitatively in this work, we would like to examine here some qualitatively important differences between quark-novae (assuming they power the decompression) and NSMs or type II SNe in relation to present observations of chemical abundances.

\subsection{QNe versus NSMs}

Failure of NSE: The QN and NSM scenarios both utilize decompression of neutron matter for the r-process. In case of NSMs, the ejected blobs of neutron matter are located in the long spiral arms generated by the merger, while the QN ejecta is assumed to flow outwards from the dense surface of a neutron star. Consequently, the density drops faster in case of NSMs than in the QN scenario, and as a result, $\beta$-decays are more effective at heating up the material to nuclear statistical equilibirium (NSE) temperatures (Freiburghaus et al. 1999b).

In the QN case, the slower expansion of the ejecta implies that $\beta$-decays happen at higher densities (significant number of $\beta$ decays occur when the $\beta$-decay timescale becomes comparable to the expansion timescale) so that the temperature rises at most to $T_{9} \sim 0.1$ (see Figs. 5 and 6 ) and NSE does not hold.

r-process yield: The r-process yield from QNe are consistent with the total r-process yield in the Galaxy $\left(\approx 10^{4} M_{\odot}\right.$, Wallerstein et al. 1997). Assuming that the phase transition to $(u, d, s)$ matter happens due to spin-down of rotating neutron stars and accretion from the ISM, statistics on pulsar velocities imply that $\mathrm{QNe}$ occur at a rate of 
$f_{\mathrm{QN}} \approx 10^{-4}-10^{-6} \mathrm{yr}^{-1} \mathrm{Galaxy}^{-1}$ (Yasutake et al. 2004) ${ }^{5}$. Each QN event can release anywhere from $M_{r}=0.001-0.1 M_{\odot}$ of baryonic matter for r-processing, therefore $M_{\mathrm{tot}} \sim f_{\mathrm{QN}} M_{r} t_{\mathrm{age}}$ is certainly in the range of our Galaxy's total r-abundance, given its age $t_{\text {age }} \sim 10^{10}$ yr.

The coalescence rate for binary neutron stars is estimated to lie in the range $3 \times 10^{-4}-10^{-6} / \mathrm{y}$. Simulations of NSMs predict that, on average, about $10^{-2} M_{\odot}$ of baryonic matter is ejected per event. Once again, this is consistent with our Galaxy's total r-abundance. The underproduction of elements at $A<130$ is a common feature between the two scenarios, but this depletion is more pronounced in NSMs, since not all neutrons are consumed in the QN.

Timescales: QNe can occur over widely different timescales. Once the central pressure inside a neutron star reaches a critical pressure, nuclear matter is in a metastable state where a droplet of quark matter is likely to be formed. This process is not immediate, however, since quantum nucleation must take into account the surface and curvature energy costs in making a quark matter bubble (Bombaci et al. 2004). The formation of such a bubble with a critical radius determined by minimizing the Gibbs free energy can be described by a tunneling process, whose probability is exponentially sensitive to the central pressure. Furthermore, the nucleation time is then given by

$\tau=\left(v_{0} p_{0} N_{\mathrm{c}}\right)^{-1}$

where $v_{0}=10^{23} / \mathrm{s}$ is the number of collisions made by a subcritical (virtual) droplet of quark matter confined in a potential well, $p_{0}$ is the tunneling probability and $N_{\mathrm{c}}=10^{48}$ is the number of droplet centers in the star's center (Iida \& Sato 1997). Results from Bombaci et al. (2004) show that the nucleation time can vary by several orders of magnitude from $1 \mathrm{yr}$ to Hubble times for small $(\sim 1 \%)$ changes in stellar mass (and hence pressures, for a given equation of state).

Coalescence timescales for neutron star mergers in the majority of binary systems range from 100-1000 Myr, although several recent works argue for a significant population of short lived binaries with merger times less than 1 Myr (Ivanova et al. 2003; Dewi \& Pols 2003; Kim et al. 2003). This puts them at odds with the observed r-enhancement in some ultra-metal poor halo stars. This would not be a problem for QNe, which could happen in the very early stages of the universe's history since the phase transition can happen even within a year of forming a neutron star in a supernova event.

Chemical inhomogeneities: As argued previously, NSMs are thought to be disfavored as dominant astrophysical sites for the r-process since they would lead to a sudden and late r-enrichment of the interstellar medium (ISM), leading to chemical inhomogenities that are inconsistent with the observed scatter in [r/Fe] of $0.2-0.3$ dex (for possible caveats to this argument, see Rosswog et al. 2000). The underlying problem is the large mass ejection in NSMs (hydrodynamic simulations indicate that up to $10^{-3}-10^{-2} M_{\odot}$ of r-process matter might be ejected in a merger event (Janka et al. 1999; Rosswog et al. 2004; Rosswog et al. 1999, 2000)) as well as the low NSM rate

\footnotetext{
5 The large range reflects the uncertainty in the high density equation of state, which is poorly known. For example, in Eriguchi et al. (2004), the bag constant $B$ for quark matter is varied from $B=$ 60-120 $\mathrm{MeV} \mathrm{fm}^{-3}$. This affects the critical density at which the phase transition happens.
}

(1-100 $\mathrm{Myr}^{-1} \mathrm{Galaxy}^{-1}$, with the upper limit being preferred). If we adopt the results of Fryer \& Woosley (1998) on mass ejection from phase transitions, the QN can eject as much material as an NSM, and their event frequency is also comparable. So, can the QN evade the problems faced by NSMs?

If $\mathrm{QNe}$ are the primary site of the r-process, the constraints from metal-poor stars are difficult to satisfy. One would have to arrange for differing effective contributions to the r-process from QNe today than in the early history of the Galaxy. This might happen in the following way: early in Galactic history, the distribution of gas (and therefore star formation) was more isotropic, so that the r-process material from QNe was absorbed into the next generation of stars. More recently, since most of the gas and star-forming regions came to reside in the Galactic disk, type II SNe go off mainly in the disk. The neutron stars thus created, on account of pulsar kicks, accquire large peculiar velocities ${ }^{6}$, with transverse speeds ranging from 0 to $\sim 1500 \mathrm{~km} \mathrm{~s}^{-1}$. This implies a mean three-dimensional speed of $450 \pm 90 \mathrm{~km} \mathrm{~s}^{-1}$ (Lyne \& Lorimer 1994), hence some of these run-away pulsars could cover kpc distances from their origins within a Hubble time before they undergo a QN explosion as a result of an increase of their core densities following spin down or accretion from the ISM. Therefore, we expect that a percentage of the total r-nuclei mass contributed by relatively recent QNe to reside in the diffuse gas around the halo of our Galaxy. Since the halo is not an active star-forming region, the r-process ejecta is not absorbed into young stars, mitigating the problem of large chemical inhomogeneities created by QNe.

It remains to perform a chemical evolution study of the enriched ISM from QNe events, and correlate the results with the observed scatter in elemental abundances. This is a further step in the modeling that will test the feasibility of the QN as an r-process site. At this stage, a detailed hydrodynamical description of the QN is lacking, and so a self-consistent analysis of chemical evolution cannot be performed; however, it is encouraging that the r-process yields calculated in this paper reveal the $\mathrm{QN}$ to be a candidate for an r-process site. Since the frequency of QNe in the Galaxy is similar to that of NSMs, we prefer to think of them as additional r-process sites to $\mathrm{SNe}$, or even as substitutes for some failed/delayed $\mathrm{SN}$ events.

\subsection{QNe versus type II SNe}

Yields and timescales: QNe can synthesize significantly larger amounts of r-process material than the typical $10^{-6}-10^{-5} M_{\odot}$ thought to be ejected in each core-collapse SN event (Cowan et al. 1991; Woosley et al. 1994; Qian 2000; Wanajo et al. 2001). The frequency of $\mathrm{QNe}$ is less than that of $\mathrm{SNe}$ $\left(10^{-2} \mathrm{yr}^{-1} \mathrm{Galaxy}^{-1}\right)$. Several of the uncertainties that plague the SN scenario also apply to the QN. The correlation of r-process yields in $\mathrm{SNe}$ with progenitor mass is a complex problem, while the size and density of the quark core in QNe is a crucial factor controlling the ejected mass fraction.

Role of neutrinos: Neutrino propagation and absorption rates in hot and dense quark matter are not known as accurately as required, a problem that is exacerbated by the fact that even the ground state of quark matter at high density is uncertain ${ }^{7}$. The

\footnotetext{
6 It is a well known fact that pulsars in our Galaxy have velocities much in excess of ordinary stars (Harrison et al. 1993).

7 In the QN, we assume it to be three-flavors of free massless quarks. In reality, a transition to a gapped phase is likely, which can drastically alter the neutrino rates.
} 
role of neutrinos in $\mathrm{SNe}$ and QNe is different, although the energy released in the form of neutrinos from core-collapse (in the case of $\mathrm{SNe}$ ) and the phase transition (in QNe) is about the same.

In $\mathrm{SNe}$, neutrino-heating at the surface of the neutron star is responsible for the neutrino-driven wind mechanism, and neutrinos continue to be important for the r-process, especially in driving fission of progenitor nuclei above $A \geq 190$ towards lighter nuclei above and below $A \sim 130$. Neutrinos can also induce neutron emission accounting for the solar r-abundances at $A=183-187$ from the progenitor peak at $A=195$, and alter the neutron-to-seed nuclei ratio (Haxton et al. 1997).

In QNe, neutrinos deposit their energy within the neutron star, and emerge as a neutrino burst only during the latter stages of r-processing. This justifies our neglect of neutrinos in the reaction network and their impact on the $Y_{\mathrm{e}}$ of the ejected material during the initial stages of expansion. However, neutrino-induced effects as stated above will also be important in determining the final yield from a $\mathrm{QN}$.

Compact remnants: An intriguing difference is that the compact remnant of type II SNe are neutron stars, whereas a QN will give birth to a quark star. If $\mathrm{QNe}$ do indeed occur in the universe, quark stars should abound, though not as much as neutron stars. A rough estimate of their number can be obtained by noting that our Galaxy likely contains about $10^{8}$ neutron stars, so that assuming QNe to be a primary site of the r-process, the constraint on the total r-process material in the Galaxy implies that 1 out of every 1000 neutron stars has undergone a QN. Since a QN leaves behind a quark star, this means that out of the approximately 1500 neutron stars observed so far, one or two may actually be quark stars. If QNe are only a secondary site, however, the above estimate for the number of quark stars may be highly optimistic, explaining why we have not detected any quark star as yet. This points to the need to distinguish clearly between neutron and quark stars, a distinction that can perhaps only be made by detailed observations of surface luminosities (Page \& Usov 2002). While stellar mass and particularly radius measurements are at present not accurate enough to differentiate neutron and quark stars (Alford et al. 2006b), recent studies of the thermal and non-thermal emission properties of the surface of bare quark stars point to super-Eddington luminosities of gamma-ray photons and $e^{+} e^{-}$pair-winds as a unique and identifiable signature (Aksenov et al. 2004; Jaikumar et al. 2004). However, the distinction may be undermined if quark stars have a crust (Jaikumar et al. 2005; Alford et al. 2006a). The signature of a QN with a fall-back crust was recently examined by Niebergal et al. (2006). The most direct test of the QN association to the r-process would come from $\gamma$ - decays of unstable nuclei produced in the r-process, as explained below.

$\gamma$-decay of r-nuclei: We expect the QN ejecta to achieve $\gamma$-ray transparency sooner than SN ejecta since QN progenitors (i.e., neutron stars) lack extended atmospheres, so that r-process only nuclei with $\gamma$-decay lifetimes of the order of years (such as ${ }^{137} \mathrm{Cs}$, ${ }^{144} \mathrm{Ce},{ }^{155} \mathrm{Eu}$ and ${ }^{194} \mathrm{Os}$ ) can be used as tags for the QN. For example, given the r-process abundance of the above nuclei in the QN ( $Y_{\mathrm{e}}=0.03, \alpha=0.0001,1 / 2$ heating), we can estimate the $\gamma$-flux as (Qian et al. 1998)

$$
\begin{aligned}
\frac{F_{\gamma}}{10^{-7} \gamma \mathrm{cm}^{-2} \mathrm{~s}^{-1}}= & 32 \times I_{\gamma} \\
& \times \frac{\delta M}{10^{-7} M_{\odot}} \frac{100}{A} \frac{1 \mathrm{yr}}{\tau}\left(\frac{10 \mathrm{kpc}}{d}\right)^{2}
\end{aligned}
$$

where $I_{\gamma}$ is the number of monochromatic photons per decay of the decaying nucleus with mass number $A$ and lifetime $\tau$, and $d$ is the distance to the QN. $\delta M$ is the expected mass of the $\gamma$-active r-process nucleus produced in a $\mathrm{QN}$, which is estimated by assuming that a total of $10^{-2} M_{\odot}$ of r-process material is ejected per QN event. For the nuclei mentioned above, the value of $F_{\gamma}$ (in the units indicated by Eq. (6)) is tabulated below for QNe, and compared to estimates from $\mathrm{SNe}$ (values taken from Qian et al. 1998):

\begin{tabular}{cccccc}
\hline \hline$F_{\gamma}$ & ${ }^{137} \mathrm{Cs}$ & ${ }^{144} \mathrm{Ce}$ & ${ }^{155} \mathrm{Eu}$ & ${ }^{194} \mathrm{Os}$ & ${ }^{126} \mathrm{Sn}$ \\
\hline QN & 0.45 & 2.0 & 0.92 & 1.5 & 0.30 \\
$\mathrm{SN}$ & 0.35 & 2.9 & 0.50 & 1.6 & 5.0 \\
\hline
\end{tabular}

In the above table, for cases where a nucleus displays several gamma-ray transitions, we chose the one with the largest flux. For each of the short-lived nuclides (all except ${ }^{126} \mathrm{Sn}$ ), we chose a typical distance of $d \sim 10 \mathrm{kpc}$ for the r-process source. For the case of ${ }^{126} \mathrm{Sn}$, which is a long-lived nucleus $(\tau=1.44 \times$ $10^{5} \mathrm{yr}$ ), only fluxes from nearby sources would be detectable, therefore, we chose $d \sim 200 \mathrm{pc}$. Even so, the flux from ${ }^{126} \mathrm{Sn}$ decay is small, since the QN is not effective at producing elements under $A \sim 130$. We note that the line sensitivities implied by these fluxes are somewhat below the detection threshold capabilities of the spectrograph (SPI) aboard the INTEGRAL satellite (Knodlseder 2002). Further, given the remaining lifetime of the INTEGRAL mission, and the rarity of QNe, it is advisable to look at $\gamma$-decays of long-lived radioactive nuclei such as ${ }^{126} \mathrm{Sn}$. However, unless a QN has occured in our vicinity $(d \leq 50 \mathrm{pc})$ recently, gamma-ray fluxes from ${ }^{126} \mathrm{Sn}$ decay would be too small to detect. We do not address any possible connection with diffuse $\gamma$-emission at the galactic center since the frequency of QNe is quite small compared to the lifetime of ${ }^{126} \mathrm{Sn}$. The gamma decay of certain radioactive nuclides beyond ${ }^{208} \mathrm{Bi}$ can also provide detectable signatures of an r-process site, provided the r-process event occurred nearby $(d \sim 0.2-1 \mathrm{kpc})$ and recently $(t \leq 700 \mathrm{yr})$. Expected fluxes from some promising nuclei identified in previous works (Clayton \& Craddock 1965; Qian et al. 1999) are calculated for a QN and the results are tabulated below, along with comparisons to the flux from a $\mathrm{SN}$ event.

\begin{tabular}{ccccc}
\hline \hline$F_{\gamma}$ & ${ }^{226} \mathrm{Ra}$ & ${ }^{229} \mathrm{Th}$ & ${ }^{227} \mathrm{Ac}$ & ${ }^{228} \mathrm{Ra}$ \\
\hline $\mathrm{QN}$ & 1.7 & 0.34 & 1.42 & 3.24 \\
$\mathrm{SN}$ & 1.0 & 0.21 & 1.2 & 3.8 \\
\hline
\end{tabular}

\section{Conclusion}

The r-process remains a complex nucleosynthetic process, requiring extensive modeling of the astrophysics as well as nuclear aspects. One of the underlying difficulties is that we are yet to identify the astrophysical site in which ideal r-process conditions are met. In this work we suggest that decompressing neutron star matter, triggered by an explosive phenomenon like a quark-nova may be a feasible site. Decompressing neutron star matter provides a rich supply of exotic nuclei and large neutron flux, and generates an r-process environment that is similar, though not identical, to neutron star mergers. We performed network calculations adapted to the study of the decompression and the subsequent r-process, and found that final r-process yields and abundance patterns are determined primarily by dynamical timescales characteristic of a quark-nova (or the underlying motive force behind the decompression), and by the extent of heating from nuclear reactions. In the context of reproducing the observed pattern of solar abundances, the slowest expansions are 
the most promising. Rapid expansions, with or without nuclear heating, lead to a prominent 2 nd peak but do not resemble the solar pattern. As a candidate for the r-process site, quark-novae can possibly evade some of the constraints from abundance observations of r-process enhanced metal-poor stars that seem to disfavor neutron star mergers as primary r-process candidates. On the basis of a preliminary exploration of the r-process yields, and qualitative features of the quark nova, we find it to be a promising scenario, to be developed and examined in more detail. A numerical study of the dynamics of the quark-nova needs to be performed to relate the mode of burning of the nuclear-quark conversion front to the expansion velocity of the ejecta; here we have only explored a plausible regime of expansion timescales. Finally, in our picture, quark-novae are intimately connected with the birth of strange stars, so we suggest that decays of $\gamma$ active r-process nuclei be used as a tag for strange stars. This intriguing link between strange stars and r-process nucleosynthesis might be confirmed by the next generation of gamma-ray satellites.

Acknowledgements. The authors are grateful to Jim Truran, Amanda Karakas and Ken Nollett for comments on the text. P.J. acknowledges helpful discussions with Lee Sobotka and Mark Alford, and support for this work by the Department of Energy (DoE), Office of Nuclear Physics, contract no. W-31-109-ENG-38 and US DoE grant DE-FG02-93ER40756. R.O is supported by an operating grant from the Natural Research Council of Canada (NSERC) as well as the Alberta Ingenuity Fund (AIF). K.O. is supported by the National Science Foundation under grant PHY 02-16783 for the Physics Frontier Center Joint Institute for Nuclear Astrophysics (JINA). B. S. M. is supported by the Department of Energy through SciDAC and by grants from NASA's cosmochemistry program.

\section{References}

Aksenov, A. G., Milgrim, M., \& Usov, V. V. 2004, ApJ, 609, 363

Alcock, C., Farhi, E., \& Olinto, A. V. 1986, ApJ, 310, 261

Alford, M. G., Rajagopal, K., Reddy, S., \& Steiner, A. W. 2006a, Phys. Rev. D, 73,114016

Alford, M. G., Blaschke, D., Drago, A., et al. $2006 \mathrm{~b}$ [arXiv: astro-ph/0606524]

Argast, D., Samland, M., Gerhard, O. E., \& Thielemann, F.-K. 2000, A\&A, 356, 873

Argast, D., Samland, M., Thielemann, F.-K., \& Qian, Y.-Z. 2004, A\&A, 416, 997

Baym, G., Bethe, H. A., \& Pethick, C. J. 1971a, Nucl. Phys. A, 175, 225

Baym, G., Pethick, C., \& Sutherland, P. 1971b, ApJ, 170, 299

Belczynski, K., Kalogera, V., \& Bulik, T. 2002, ApJ, 572, 407

Bodmer, A. R. 1971, Phys. Rev. D, 4, 1601

Bombaci, I., Parenti, I., \& Vidana, I. 2004, ApJ, 614, 314

Burbidge, G. R., Burbidge, W. A., Fowler, W. A., \& Hoyle, F. 1957, Rev. Mod. Phys., 29, 547

Cameron, A. G. W. 1957, PASP, 69, 201

Cardall, C. Y., \& Fuller, G. M. 1997, ApJ, 486, L111

Cheng, K. S., \& Dai, Z. G. 1996, Phys. Rev. Lett., 77, 1210

Clayton, D. D., \& Craddock, W. L. 1965, ApJ, 142, 189

Cowan, J. J., Thielemann, F.-K., \& Truran, J. W. 1991, Phys. Rep., 208, 267

Cowan, J. J., et al. 2002, ApJ, 572, 861

Dewi, J. D. M., \& Pols, O. R. 2003, MNRAS, 344, 629

Drago, A., Lavagno, A., \& Parenti, I. 2005 [arXiv: astro-ph/0512652]

Farhi, E., \& Jaffe, R. L. 1984, Phys. Rev. D, 30, 2379

Freiburghaus, C., Rembges, F., Rauscher, T., et al. 1999a, ApJ, 516, 381

Freiburghaus, C., Rosswog, S., \& Thielemann, F.-K. 1999b, ApJ, 525, L121

Fryer, C. L., \& Woosley, S. E. 1998, ApJ, 501, 780

Fryer, C. L., Woosley, S. E., \& Hartmann, D. H. 1999, ApJ, 526, 152

Gentile, N. A., Aufderheide, M. B., Mathews, G. J., Swesty, F. D., \& Fuller, G. M. 1997, ApJ, 414, 701

Goriely, S., Demetriou, P., Janka, H.-T., Pearson, J. M., \& Samyn, M. 2004, Nucl. Phys. A, 758, 587

Harrison, P. A., Lyne, A. G., \& Anderson, B. 1993, MNRAS, 261, 113

Haxton, W. C., Langanke, K., Qian, Y.-Z., \& Vogel, P. 1997, Phys. Rev. Lett., 78,2694
Hill, V., et al. 2002, A\&A, 387, 560

Horvath, J. E., \& Benvenuto, O. G. 1988, Phys. Lett. B, 213, 516

Iida, K., \& Sato, K. 1997, Prog. Theor. Phys., 98, 277

Ishimaru, Y., \& Wanajo, S. 1999, ApJ, 511, L33

Ishimaru, Y., Wanajo, S., Aoki, W., \& Ryan, S. G. 2004, ApJ, 600, L47

Itoh, N. 1970, Prog. Theor. Phys., 44, 291

Ivanova, N., Belczynski, K., Kalogera, V., Rasio, F. A., \& Taam, R. E. 2003, ApJ, 592, 475

Jaikumar, P., Gale, C., Page, D. P., \& Prakash, M. 2004, Phys. Rev. D, 70, 023004 Jaikumar, P. Reddy, S., \& Steiner, A. W. 2005, Phys. Rev. Lett., 96, 041101 Janka, H.-T., Eberl, T., Ruffert, M., \& Fryer, C. L. 1999, ApJ, 527, L39 Jordan, G. C., \& Meyer, B. S. 2004, ApJ, 617, L131

Kappeler, F., Beer, H., \& Wisshak, K. 1989, Rep. Progr. Phys., 52, 945

Kim, C., Kalogera, V., \& Lorimer, D. R. 2003, AIP Conf. Proc., 686, 281

Knodlseder, J. 2002, New Views on MICROQUASARS, ed. Ph. Durouchoux, Y. Fuchs, \& J. Rodriguez (Kolkata, India: Center for Space Physics), 385 Keränen, P., \& Ouyed, R. 2003, A\&A, 407, L51

Keränen, P., Ouyed, R., \& Jaikumar, P. 2005, ApJ, 618, 485

Lattimer, J. M., \& Schramm, D. N. 1974, ApJ, 192, L145

Lattimer, J. M., \& Swesty, D. 1991, Nuc. Phys. A, 535, 331

Lattimer, J. M., Mackie, F., Ravenhall, D. G., \& Schramm, D. N. 1977, ApJ, 213, 225

Lorenz, C. P., Ravenhall, D. G., \& Pethick, C. J. 1993, Phys. Rev. Lett., 70, 4, 379

Lyne, A., \& Lorimer, D. R. 1994, Nature, 369, 127

Lugones, G., Benvenuto, O. G., \& Vucetich, H. 1994, Phys. Rev. D, 50, 6100

Marranghello, G. F., \& de Freitas Pacheco, J. A. 2005, Int. J. Mod. Phys. D, 15, 153

Mazzali, P. A., \& Chugai, N. N. 1995, A\&A, 303, 118

Meyer, B. S. 1989, ApJ, 343, 254

Meyer, B. S., \& Schramm, D. N. 1988, Origin and Distribution of the Elements, ed. G. J. Mathews (Singapore: World Scientific Publishing), 610

Meyer, B. S., \& Howard, W. M. 1991, in Supernovae, ed. S. E. Woosley (New York: Springer-Verlag), 630

Meyer, B. S., \& Brown, J. S. 1997, ApJS, 112, 199

Olesen, M. J., \& Madsen, J. 1991, Nucl. Phys. B, Proc. Suppl., 24, 170

Otsuki, K., Tagoshi, H., Kajino, T., \& Wanajo, S. 2000, ApJ, 533, 424

Ouyed, R., Dey, J., \& Dey, M. 2002, A\&A, 390, L39

Ouyed, R., Leahy, D., \& Niebergal, D. 2006 [arXiv: astro-ph/0608536]

Page, D. P., \& Usov, V. V. 2002, Phys. Rev. Lett., 89, 131101

Portegies, Z. S. F., \& Yungel'son, L. R. 1998, A\&A, 332, 173

Prakash, M., Bombaci, I., Prakash, M., et al. 1997, Phys. Rep., 280, 1

Qian, Y.-Z. 2000, ApJ, 534, L67

Qian, Y.-Z. 2003, Prog. Part. Nucl. Phys., 50, 153

Qian, Y.-Z. 2004, in The r-process: The Astrophysical Origin of the Heavy Elements and Related Rare Isotope Accelerator Physics, ed. Y.-Z. Qian, E. Rehm, H. Schatz, \& F.-K. Thielemann (Singapore: World Scientific), 157

Qian, Y.-Z., \& Woosley, S. E. 1996, ApJ, 471, 331

Qian, Y.-Z., Vogel, P., \& Wasserburg, G. J. 1998, ApJ, 506, 868

Qian, Y.-Z., Vogel, P., \& Wasserburg, G. J. 1999, ApJ, 524, 213

Rosswog, S., Liebendorfer, M., Thielemann, F.-K., et al. 1999, A\&A, 341, 499

Rosswog, S., Freiburghaus, C., \& Thielemann, F.-K. 2001, Procceedings of Nuclei in the Cosmos 2000, Nucl. Phys. A, 688, 344

Rosswog, S., Speith, R., \& Wynn, G. A. 2004, MNRAS, 351, 1121

Sneden, C., McWilliam, A., Preston, G. W., et al. 1996, ApJ, 467, 819

Sneden, C., Cowan, J. J., Lawler, J. E., et al. 2003, ApJ, 591, 936

Staff, J. E., Ouyed, R., \& Jaikumar, P. 2006, ApJ, 645, L145

Sumiyoshi, K., Yamada, S., Suzuki, H., \& Hillebrandt, W. 1998, A\&A, 334, 159

Sumiyoshi, K., Suzuki, K., Otsuki, K., Terasawa, M., \& Yamada, S. 2000, Pub. Astron. Soc. Japan, 52, 60

Sumiyoshi, K., Terasawa, M., Mathews, G. J., et al. 2001, ApJ, 562, 880

Surman, R., McLaughlin, G. C., \& Hix, W. R. 2006, ApJ, 643, 1057

Symbalisty, E., \& Schramm, D. N. 1982, ApJ, 22, 143

Takahashi, K., Witti, J., \& Janka, H.-T. 1994, A\&A, 286, 857

Thompson, T. A., Burrows, A., \& Meyer, B. S. 2001, ApJ, 562, 887

Truran, J. W., Cowan, J. J., Pilachowski, C. A., \& Sneden, C. 2002, PASP, 114, 1293

Wallerstein, G., Iben, I. Jr., Parker, P., et al. 1997, Rev. Mod. Phys., 69, 995

Wanajo, S., Toshitaka, K., Mathews, G. J., \& Otsuki, K. 2001, ApJ, 554, 578

Wanajo, S., Naoki, I., Ishimaru, Y., et al. 2002, ApJ, 577, 853

Wanajo, S., Tamamura, M., \& Naoki, I., et al. 2003, ApJ, 593, 968

Witten, E. 1984, Phys. Rev. D, 30, 272

Woosley, S. E., Wilson, J. R., Mathews, G. J., et al. 1994, ApJ, 433, 229

Yasutake, N., Hashimoto, M.-A., \& Eriguchi, Y. 2004, Prog. Theor. Phys., 113, 